\title{
SOME SUBGROUPS OF THE CREMONA GROUPS
}

\author{
VLADIMIR L. POPOV*
}

To M. Miyanishi on his 70 th birthday

\begin{abstract}
We explore algebraic subgroups of the Cremona group $\mathcal{C}_{n}$ over an algebraically closed field of characteristic zero. First, we consider some class of algebraic subgroups of $\mathcal{C}_{n}$ that we call flattenable. It contains all tori. Linearizability of the natural rational actions of flattenable subgroups on $\mathbf{A}^{n}$ is intimately related to rationality of the invariant fields and, for tori, is equivalent to it. We prove stable linearizability of these actions and show the existence of nonlinearizable actions among them. This is applied to exploring maximal tori in $\mathcal{C}_{n}$ and to proving the existence of nonlinearizable, but stably linearizable elements of infinite order in $\mathcal{C}_{n}$ for $n \geqslant 5$. Then we consider some subgroups $\mathcal{J}\left(x_{1}, \ldots, x_{n}\right)$ of $\mathcal{C}_{n}$ that we call the rational de Jonquières subgroups. We prove that every affine algebraic subgroup of $\mathcal{J}\left(x_{1}, \ldots, x_{n}\right)$ is solvable and the group of its connected components is Abelian. We also prove that every reductive algebraic subgroup of $\mathcal{J}\left(x_{1}, \ldots, x_{n}\right)$ is diagonalizable. Further, we prove that the natural rational action on $\mathbf{A}^{n}$ of any unipotent algebraic subgroup of $\mathcal{J}\left(x_{1}, \ldots, x_{n}\right)$ admits a rational cross-section which is an affine subspace of $\mathbf{A}^{n}$. We show that in this statement "unipotent" cannot be replaced by "connected solvable". This is applied to proving a conjecture of A. Joseph on the existence of "rational slices" for the coadjoint representations of finite-dimensional algebraic Lie algebras $\mathfrak{g}$ under the assumption that the Levi decomposition of $\mathfrak{g}$ is a direct product. We then consider some overgroup $\widehat{\mathcal{J}}\left(x_{1}, \ldots, x_{n}\right)$ of $\mathcal{J}\left(x_{1}, \ldots, x_{n}\right)$ and prove that every torus in $\widehat{\mathcal{J}}\left(x_{1}, \ldots, x_{n}\right)$ is linearizable. Finally, we prove the existence of an element $g \in \mathcal{C}_{3}$ of order 2 such that $g \notin G$ for every connected affine algebraic subgroup $G$ of $\mathcal{C}_{\infty}$; in particular, $g$ is not stably linearizable.
\end{abstract}

\section{INTRODUCTION}

The last three decades were marked by growing interest in problems related to the affine Cremona group $\mathcal{C}_{n}^{\text {aff }}$ (the group of biregular automorphisms of the affine $n$-dimensional space $\mathbf{A}^{n}$ ). Despite of a remarkable progress made during these years, some fundamental problems still remain unsolved. For instance, at the moment the linearization problem for algebraic tori is solved only for $n \leqslant 3$ and its difficult solution for $n=3$ is one of the highlights of the theory.

\footnotetext{
* Supported by grants РФФИ 11-01-00185-a, НШ-5139.2012.1, and the program Contemporary Problems of Theoretical Mathematics of the Russian Academy of Sciences, Branch of Mathematics.
} 
Some of these problems may be formulated entirely in terms of grouptheoretic structure of $\mathcal{C}_{n}^{\text {aff }}$. Thereby, they admit the birational counterparts related to the full Cremona group $\mathcal{C}_{n}$ (the group of birational automorphisms of $\mathbf{A}^{n}$ ). It is of interest to explore them. We have not seen publications purposefully developing this viewpoint. A step in this direction is made in this paper.

In Section 2 we first consider a class of algebraic subgroups of $\mathcal{C}_{n}$ that we call flattenable. Linearizability of their natural rational actions on $\mathbf{A}^{n}$ is intimately related to rationality of their invariant fields, the subject of classical Noether problem. All algebraic tori in $\mathcal{C}_{n}$ are contained in this class and, for them, these two properties, linearizability and rationality, are equivalent. We show that flattenable groups are special in the sense of Serre (see [Ser58]) and that every rational locally free action on $\mathbf{A}^{n}$ of a special group is stably linearizable; in particular, this is so for tori. On the other hand, we show that there are stably linearizable, but nonlinearizable rational locally free actions on $\mathbf{A}^{n}$ of connected affine algebraic groups, in particular, that of tori. We then apply this to the problem of describing maximal tori in $\mathcal{C}_{n}$ and show that nowadays one can say more on it than in the time when BiałynickiBirula and Demazure wrote their papers [Bia66], [Dem70]. Namely, apart from $n$-dimensional maximal tori (that are all conjugate), $\mathcal{C}_{n}$ for $n \geqslant 5$ contains maximal tori of dimension $n-3$ (and does not contain maximal tori of dimensions $n-2, n-1$ and $>n$ ). This answers a question of Hirschowitz in [Hir72, Sect. 3]. As another application, we prove the existence of nonlinearizable, but stably linearizable elements of infinite order in $\mathcal{C}_{n}$ for $n \geqslant 5$.

In Sections 3 and 4 we consider a natural counterpart of the classical de Jonquières subgroups of $\mathcal{C}_{n}^{\text {aff }}$ that we call the rational de Jonquières subgroups of $\mathcal{C}_{n}$. We prove that their affine algebraic subgroups are solvable and have Abelian groups of connected components. We also prove that reductive algebraic subgroups of the rational de Jonquières subgroups of $\mathcal{C}_{n}$ are diagonalizable. Then we prove that for the natural rational action on $\mathbf{A}^{n}$ of any unipotent algebraic subgroup of a rational de Jonquières subgroup of $\mathcal{C}_{n}$ there exists an affine subspace of $\mathbf{A}^{n}$ which is a rational cross-section for this action (recall that for rational actions of connected solvable affine algebraic subgroups of $\mathcal{C}_{n}$ on $\mathbf{A}^{n}$, the existence of some rational cross-sections, not necessarily affine subspaces of $\mathbf{A}^{n}$, is ensured by the general Rosenlicht's theorem, see [Ros56, Theorem 10]). We also show that in this result "unipotent" cannot be replaced by "connected solvable". We then apply this result to a conjecture of A. Joseph ([Jos11, Sect. 7.11]) on the existence of "rational slices" for the coadjoint representations of finite-dimensional algebraic Lie algebras $\mathfrak{g}$ and prove this conjecture under the assumption that the Levi decomposition of $\mathfrak{g}$ is a direct product. Further, we consider a certain natural class of overgroups of the rational de Jonquières subgroups and, using the results of Section 2, show that the natural action on $\mathbf{A}^{n}$ of any subtorus of such an overgroup is linearizable. Finally, we prove the existence of an element $g \in \mathcal{C}_{3}$ of order 2 such that $g \notin G$ for every connected affine algebraic subgroup $G$ of the direct limit $\mathcal{C}_{\infty}$ of the tower of natural inclusions $\mathcal{C}_{1} \hookrightarrow \mathcal{C}_{2} \hookrightarrow \cdots ;$ in particular, $g$ is not stably linearizable. 


\section{Conventions, notation and some generalities}

Below "variety" means "algebraic variety". We assume given an algebraically closed field $k$ of characteristic zero which serves as domain of definition for each of the varieties considered below. Each variety is identified with its set of points rational over $k$. Along with the standard notation and conventions of [Bor91] we use the following ones.

- Aut $X$ is the automorphism group of a variety $X$.

- Bir $X$ is the group of birational automorphisms of an irreducible variety $X$.

$-X \approx Y$ means that $X$ and $Y$ are birationally isomorphic irreducible varieties.

- If $f$ is a rational function on the product $X \times Y$ of varieties and $x \in X$ is a point such that $\left.f\right|_{x \times Y}$ is well defined, then $f(x)$ is the element of $k(Y)$ such that $f(x, y)=f(x)(y)$ for every point $(x, y) \in X \times Y$ where $f$ is defined.

- Given a dominant rational map $\varphi: X \rightarrow Y$ of varieties, $\varphi^{*}$ is the embedding $k(Y) \hookrightarrow k(X), f \mapsto f \circ \varphi$.

- Given an action

$$
\alpha: G \times X \rightarrow X
$$

of a group $G$ on a set $X$ and the elements $g \in G, x \in X$, then $\alpha(g, x) \in X$ is denoted by $g \cdot x$. If $H$ is a subgroup of $G$, then $\left.\alpha\right|_{H}$ is the restriction of $\alpha$ to $H \times X$.

- $\mathbf{A}^{n} \times \mathbf{A}^{m}$ is identified with $\mathbf{A}^{n+m}$ by means of the isomorphism

$$
\begin{aligned}
\mathbf{A}^{n} \times \mathbf{A}^{m} & \rightarrow \mathbf{A}^{n+m}, \\
\left(\left(a_{1}, \ldots, a_{n}\right),\left(b_{1}, \ldots, b_{m}\right)\right) & \mapsto\left(a_{1}, \ldots, a_{n}, b_{1}, \ldots, b_{m}\right) .
\end{aligned}
$$

- $K^{\times}$is the multiplicative group of a field $K$.

- $K^{+}$is the additive group of a field $K$.

- If $K / F$ is a field extension, then $K$ is called pure (resp. stably pure) over $F$ if $K$ is generated over $F$ by a finite collection of algebraically independent elements (resp. if $K$ is contained in a field that is pure over both $K$ and $F$ ).

- $G^{0}$ is the identity component of an algebraic group $G$.

— "Torus" means "affine algebraic torus".

Let $G$ be an algebraic group and let $X$ be a variety.

If (1) is a morphism, then $\alpha$ is called a regular action. In this case, for every element $g \in G$, the map $X \rightarrow X, x \mapsto g \cdot x$, is an automorphism of $X$ and the image of the homomorphism $G \rightarrow$ Aut $X, g \mapsto\{x \mapsto g \cdot x\}$ is called an algebraic subgroup of Aut $X$. A regular action $\alpha$ is called locally free if there is a dense open subset $U$ of $X$ such that the $G$-stabilizer of every point of $U$ is trivial.

From now on we assume that $X$ is irreducible. The map

$$
\operatorname{Bir} X \rightarrow \operatorname{Aut}_{k} k(X), \quad \varphi \mapsto\left(\varphi^{*}\right)^{-1},
$$


is a group isomorphism. We always identify $\operatorname{Bir} X$ and $\operatorname{Aut}_{k} k(X)$ by means of (2) when we speak about action of a subgroup of Bir $X$ by $k$-automorphisms of $k(X)$ and, conversely, action of a subgroup of $\mathrm{Aut}_{k} k(X)$ by birational automorphisms of $X$.

Let $\theta: G \rightarrow \operatorname{Bir} X$ be an abstract group homomorphism. It determines an action of $G$ on $X$ by birational isomorphisms. If the domain of definition of the partially defined map $G \times X \rightarrow X,(g, x) \mapsto \theta(g)(x)$, contains a dense open subset of $G \times X$ and coincides on it with a rational map $\varrho: G \times X \rightarrow X$, then this action (and $\varrho$ ) is called a rational action of $G$ on $X$ and $\theta(G)$ is called an algebraic subgroup of $\operatorname{Bir} X$.

There is a method for constructing algebraic subgroups of Bir $X$. Namely, let $Y$ be another irreducible variety and let $\gamma: Y \rightarrow X$ be a birational isomorphism. Then $\operatorname{Bir} Y \rightarrow \operatorname{Bir} X, g \mapsto \gamma \circ g \circ \gamma^{-1}$, is a group isomorphism and the image of any algebraic subgroup of Aut $Y$ under it is an algebraic subgroup of Bir $X$. In fact, by [Ros56, Theorem 1], this method is universal, i.e., every algebraic subgroup of $\operatorname{Bir} X$ is obtained in this manner for the appropriate $Y$ and $\gamma$. In other words, for every rational action of $G$ on $X$ there is a regular action of $G$ on an irreducible variety $Y$, the open subsets $X_{0}$ and $Y_{0}$ of resp. $X$ and $Y$, and an isomorphism $Y_{0} \rightarrow X_{0}$ such that the induced field isomorphism $k(X)=k\left(X_{0}\right) \rightarrow k\left(Y_{0}\right)=k(Y)$ is $G$-equivariant. If the action of $G$ on $Y$ is locally free, then the rational action $\varrho$ is called locally free.

Let $\varrho: G \times X \rightarrow X$ be a rational action of $G$ on $X$ and let $f$ be an element of $k(X)$. Then $\{g \cdot f \mid g \in G\}$ is an "algebraic family" of rational functions on $X$ in the following sense: there is a rational function $\widehat{f} \in k(G \times X)$ such that $g \cdot f=\widehat{f}(g)$ for every $g \in G$. Indeed, $\varrho^{*}(f) \in k(G \times X)$ and $\varrho^{*}(f)(g, x)=\left(g^{-1} \cdot f\right)(x)$ for every point $(g, x) \in G \times X$ where $\varrho^{*}(f)$ is defined; whence the claim.

If $X$ and $Y$ are irreducible varieties endowed with rational actions of $G$ such that there is a $G$-equivariant birational isomorphism $X \rightarrow Y$, then we write

$$
X \stackrel{G}{\approx} Y
$$

In order to avoid a confusion, in some cases when several rational actions are simultaneously considered, we denote $X$ endowed with a rational action $\varrho$ of $G$ by

$$
\varrho X
$$

If $Y$ is another variety, then $X \times Y$ endowed with the rational action of $G$ via the first factor by means of $\varrho$ is denoted by

$$
{ }_{\varrho} X \times Y \text {. }
$$

We denote by

$$
{ }_{\lambda} G
$$

the underlying variety of $G$ endowed with the action of $G$ by left translations.

If $\varrho$ is a rational action of $G$ on $X$, then

$$
\pi_{G, X}: X \rightarrow X_{\prime}^{\prime} G
$$


is a rational quotient of $\varrho$, i.e., $X_{i}^{\prime} G$ and $\pi_{G, X}$ are resp. a variety and a dominant rational map such that $\pi_{G, X}^{*}\left(k\left(X_{i}^{\prime} G\right)\right)=k(X)^{G}$ (see [PV94, Sect. 2.4]). Depending on the situation we choose $X_{\prime}^{\prime} G$ as a suitable variety within the class of birationally isomorphic ones. A rational section (resp., cross-section) for $\varrho$ is a rational map $\sigma: X_{\prime}^{\prime} G \rightarrow X$ such that $\pi_{G, X} \circ \sigma=$ id (resp., a subvariety $S$ of $X$ such that $\left.\pi_{G, X}\right|_{S}: S \rightarrow X_{\prime}^{\prime} G$ is a birational isomorphism). The closure of the image of a rational section is a rational cross-section and, since char $k=0$, the closure of every cross-section is obtained in this manner.

The group

$$
\mathcal{C}_{n}:=\operatorname{Aut}_{k} k\left(\mathbf{A}^{n}\right)
$$

is called the Cremona group of rank $n$ (over $k$ ). It is endowed with a topology, the Zariski topology of $\mathcal{C}_{n}$, in which families of elements of $\mathcal{C}_{n}$ "algebraically parametrized" by algebraic varieties are closed, [Ser08, Sect. 1.6]. For every algebraic subgroup $G$ of $\mathcal{C}_{n}$ and its subset $S$, the closure of $S$ in $\mathcal{C}_{n}$ coincides with the closure of $S$ in $G$ in the Zariski topology of $G$. In particular, $G$ is closed in $\mathcal{C}_{n}$. Left and right translations of $\mathcal{C}_{n}$ are homeomorphisms.

We denote by $x_{1}, \ldots, x_{n} \in k\left[\mathbf{A}^{n}\right]$ the standard coordinate functions on $\mathbf{A}^{n}$ :

$$
x_{i}\left(\left(a_{1}, \ldots, a_{n}\right)\right)=a_{i} .
$$

They are algebraically independent over $k$ and $k\left(\mathbf{A}^{n}\right)=k\left(x_{1}, \ldots, x_{n}\right)$. For every $n \geqslant 2$, we identify $\mathbf{A}^{n-1}$ with the image of the embedding $\mathbf{A}^{n-1} \hookrightarrow \mathbf{A}^{n}$, $\left(a_{1}, \ldots, a_{n-1}\right) \mapsto\left(a_{1}, \ldots, a_{n-1}, 0\right)$, and denote the restriction $\left.x_{i}\right|_{\mathbf{A}^{n-1}}$ for $i=$ $1, \ldots, n-1$ still by $x_{i}$. Correspondingly, we have the embedding $\mathcal{C}_{n-1} \hookrightarrow \mathcal{C}_{n}$, $g \mapsto \widehat{g}$, where $\widehat{g} \cdot x_{i}:=g \cdot x_{i}$ if $i=1, \ldots, n-1$ and $\widehat{g} \cdot x_{n}:=x_{n}$. The direct limit for the tower of these embeddings $\mathcal{C}_{1} \hookrightarrow \mathcal{C}_{2} \hookrightarrow \cdots \hookrightarrow \mathcal{C}_{n} \hookrightarrow \cdots$ is the Cremona group $\mathcal{C}_{\infty}$ of infinite rank. We identify every $\mathcal{C}_{n}$ with the subgroup of $\mathcal{C}_{\infty}$ by means of the natural embedding $\mathcal{C}_{n} \hookrightarrow \mathcal{C}_{\infty}$. A subgroup $G$ of $\mathcal{C}_{\infty}$ is called algebraic if there exists an integer $n>0$ such that $G$ is an algebraic subgroup of $\mathcal{C}_{n}$.

We distinguish the following two algebraic subgroups of $\mathcal{C}_{n}$ :

$$
\begin{aligned}
\mathrm{GL}_{n} & :=\left\{g \in \mathcal{C}_{n} \mid g \cdot x_{i}=\sum_{j=1}^{n} \alpha_{i j} x_{j}, \quad \alpha_{i j} \in k\right\}, \\
D_{n} & :=\left\{g \in \mathcal{C}_{n} \mid g \cdot x_{i}=\alpha_{i} x_{i}, \quad \alpha_{i} \in k\right\}
\end{aligned}
$$

$D_{n}$ is the maximal torus in $\mathrm{GL}_{n}$.

Let $g$ be an element and let $G$ be a subgroup of $\mathcal{C}_{n}$. If $g \in \mathrm{GL}_{n}$ (resp. $G \subseteq$ $\mathrm{GL}_{n}$ ), then $g$ (resp. $G$ ) is called a linear element (resp. a linear subgroup). If $g$ (resp. $G$ ) is conjugate to a linear element (resp. a linear subgroup), then it is called a linearizable element (resp. a linearizable subgroup). If $g$ (resp. $G$ ) is a linearizable element (resp. a linearizable subgroup) of some $\mathcal{C}_{m}$ for $m \geqslant n$, then it is called a stably linearizable element (resp. a stably linearizable subgroup). A rational action $\varrho$ of an algebraic group $H$ on $\mathbf{A}^{n}$ is called resp. a linear, linearizable or stably linearizable action if the image of $H$ in $\mathcal{C}_{n}$ corresponding to $\varrho$ is resp. a linear, linearizable or stably linearizable subgroup of $\mathcal{C}_{n}$. 
Acknowledgment. I am grateful to Ming-ChAng KANG who drew my attention to paper [Sh-B04].

\section{Flattening, Linearizability, tori}

Definition 1. An affine algebraic group $G$ is called flattenable if the underlying variety of $G$ endowed with the action of $G$ by left translations admits an equivariant open embedding into some $\mathbf{A}^{n}$ endowed with a rational linear action of $G$. The $G$-module $\mathbf{A}^{n}$ is then called a flattening of $G$.

Every flattenable group is connected.

Example 1. $\mathbf{A}^{n}$ endowed with the natural action of $D_{n}$,

$$
\operatorname{diag}\left(\varepsilon_{1}, \ldots, \varepsilon_{n}\right) \cdot\left(a_{1}, \ldots, a_{n}\right):=\left(\varepsilon_{1} a_{1}, \ldots, \varepsilon_{n} a_{n}\right),
$$

is a flattening of $D_{n}$. Hence, every torus is flattenable.

Example 2. The underlying vector space of the algebra $\operatorname{Mat}_{n \times n}$ of all $(n \times n)$-matrices with entries in $k$ endowed with the action of $\mathrm{GL}_{n}$ by left multiplications, $g \cdot a:=g a, g \in \mathrm{GL}_{n}, a \in \mathrm{Mat}_{n \times n}$, is a flattening of $\mathrm{GL}_{n}$. Hence, $\mathrm{GL}_{n}$ is flattenable.

Example 3. Let $G_{1}, \ldots, G_{s}$ be affine algebraic groups and let $G:=G_{1} \times$ $\cdots \times G_{s}$. If $\mathbf{A}_{n_{i}}$ endowed with an action of $G_{i}$ is a flattening of $G_{i}$, then $\mathbf{A}_{n_{1}} \times \cdots \times \mathbf{A}_{n_{s}}$ endowed with the natural action of $G$ is a flattening of $G$. Hence, $G$ is flattenable if every $G_{i}$ is.

Example 4. Consider a finite-dimensional associative (not necessarily commutative) $k$-algebra $A$ with an identity element. The group of all invertible elements of $A$ is then a connected affine algebraic group $G$ whose underlying variety is an open subset of that of $A$. The action of $G$ on $A$ by left multiplications is linear and the identity map is an equivariant embedding of $G$ into $A$. Thus, $A$ is a flattening of $G$ and $G$ is flattenable. If $A$ is the product of $n$ copies of the $k$-algebra $k$, we obtain Example 1 . Taking $A=\operatorname{Mat}_{n \times n}$, we obtain Example 2.

In general, flattening of $G$ is not unique.

Example 5. Mat ${ }_{n \times n}$ endowed with the action of $\mathrm{GL}_{n}$ given by $g \cdot a:=$ $\left(g^{t}\right)^{-1} a, g \in \mathrm{GL}_{n}, a \in \mathrm{Mat}_{n \times n}$, where $g^{t}$ is the transpose of $g$, is a flattening of $\mathrm{GL}_{n}$. It is not isomorphic to that from Example 1 (as the highest weights of these two flattenings are not equal).

Lemma 1. If the underlying variety of a connected affine reductive algebraic group $G \neq\{e\}$ is isomorphic to an open subset $U$ of $\mathbf{A}^{n}$, then $U \neq \mathbf{A}^{n}$ and the center of $G$ is at least one-dimensional.

Proof. As $G \neq\{e\}$ is reductive, it contains a torus $T$ of positive dimension. For the action of $T$ on $G$ by left translations, the fixed point set is empty. But for any regular action of $T$ on $\mathbf{A}^{n}$, the fixed point set is nonempty, see [Bia66, Theorem 1]. Hence, $U \neq \mathbf{A}^{n}$.

Since $D:=\mathbf{A}^{n} \backslash U \neq \varnothing$ and $U$ is affine, the dimension of every irreducible component of $D$ is $n-1$, see [Pop72, Lemma 3]. Since Pic $\mathbf{A}^{n}=0$, this entails 
that $D$ is the zero set of some regular function $f$ on $\mathbf{A}^{n}$. Therefore, $\left.f\right|_{U}$ is a nonconstant invertible regular function on $U$. By [Ros61 1 , Theorem 3], every such function is, up to a scalar multiple, a character of $G$. So there is a nontrivial character of $G$. On the other hand, as $G$ is a connected reductive group, $G=G^{\prime} \cdot C$ where $G^{\prime}$ is the derived group of $G$ (it is semisimple), $C$ is the connected component of the identity in the center of $G$ (it is a torus), and $G^{\prime} \cap C$ is finite, see [Bor91, Sect. 14.2]. This entails that the character group of $G$ is a free abelian group of rank $\operatorname{dim} C$. Hence, $\operatorname{dim} C \geqslant 1$.

Corollary 1. There are no nontrivial semisimple flattenable groups.

Recall from [Ser58] that an algebraic group $G$ is called special if every principal homogeneous space under $G$ over every field $K$ containing $k$ is trivial. By [Ser58] special group is automatically connected and affine. Special groups are classified: a connected affine algebraic group $G$ is special if and only if a maximal connected semisimple subgroup of $G$ is isomorphic to

$$
\mathrm{SL}_{n_{1}} \times \cdots \times \mathrm{SL}_{n_{r}} \times \mathrm{Sp}_{m_{1}} \times \cdots \times \mathrm{Sp}_{m_{s}}
$$

for some integers $r \geqslant 0, s \geqslant 0, n_{i}, m_{j}$ (by [Ser58] such groups are special, and by [Gro58] only these are).

Lemma 2. Every flattenable group $G$ is special.

Proof. Let $\mathbf{A}^{n}$ endowed with a rational linear action $\alpha$ of $G$ be a flattening of $G$ and let $\pi_{G, \alpha} \mathbf{A}^{n}:{ }_{\alpha} \mathbf{A}^{n} \rightarrow{ }_{\alpha} \mathbf{A}^{n}{ }_{\prime}^{\prime} G$ be a rational quotient for this action. By Definition 1, $\alpha$ is locally free. Hence, by [Pop94, Theorem 1.4.3], proving that $G$ is special is equivalent to proving that $\pi_{G,_{\alpha} \mathbf{A}^{n}}$ admits a rational section. But the existence of such a rational section is clear because Definition 1 entails that ${ }_{\alpha} \mathbf{A}_{i}^{n} G$ is a single point.

By Lemma 2 a maximal connected semisimple subgroup of every flattenable group is isomorphic to a group of type (5). Hence, every reductive flattenable group is a quotient $(T \times S) / C$ where $T$ is a torus, $S$ is a group of type (5) and $C$ is a finite central subgroup.

Conjecture. The following properties of a connected reductive algebraic group $G$ are equivalent:

(i) $G$ is flattenable;

(ii) $G$ is isomorphic to $\mathrm{GL}_{n_{1}} \times \cdots \times \mathrm{GL}_{n_{r}}$.

Theorem 1. Let $\alpha$ be a locally free rational action of a flattenable group $G$ on $\mathbf{A}^{m}$. If the invariant field $k\left({ }_{\alpha} \mathbf{A}^{m}\right)^{G}$ is pure over $k$, then $\alpha$ is linearizable.

Proof. Consider for $\alpha$ a rational quotient,

$$
\pi_{G, \alpha} \mathbf{A}^{m}:{ }_{\alpha} \mathbf{A}^{m} \rightarrow{ }_{\alpha} \mathbf{A}^{m} i^{\prime} G \text {. }
$$

As explained in Introduction, there is a variety $X$ endowed with a regular locally free action $\alpha^{\prime}$ of $G$ such that $\alpha^{\prime} X \stackrel{G}{\approx}{ }_{\alpha} \mathbf{A}^{m}$. By [CTKPR11, Theorem 2.13], shrinking $X$ if necessary, we may assume that the geometric quotient

$$
{ }_{\alpha^{\prime}} X \rightarrow{ }_{\alpha^{\prime}} X / G
$$


for $\alpha^{\prime}$ exists and is a torsor over $\alpha^{\prime} X / G$. As $G$ is special by Lemma 2, this torsor is locally trivial in Zariski topology. Hence, $\alpha^{\prime} X \underset{{ }_{\lambda}}{\approx} G \times\left(\alpha^{\prime} X / G\right)$ and therefore,

$$
{ }_{\alpha} \mathbf{A}^{m} \stackrel{G}{\approx}{ }_{\lambda} G \times\left({ }_{\alpha} \mathbf{A}^{m}{ }_{\prime}^{\prime} G\right)
$$

Let ${ }_{\beta} \mathbf{A}^{n}$ be a flattening of $G$. Definition 1 yields

$$
{ }_{\lambda} G \stackrel{G}{\approx}{ }_{\beta} \mathbf{A}^{n} \text {. }
$$

From (7) and (8) we obtain

$$
{ }_{\alpha} \mathbf{A}^{m} \stackrel{G}{\approx}{ }_{\beta} \mathbf{A}^{n} \times\left({ }_{\alpha} \mathbf{A}^{m} ; G\right)
$$

The assumption of purity and (9) yield

$$
{ }_{\alpha} \mathbf{A}^{m}{ }_{\prime}^{\prime} G \approx \mathbf{A}^{m-n} .
$$

Consider the action $\gamma$ of $G$ on $\mathbf{A}^{m}$ defined by

$$
{ }_{\gamma} \mathbf{A}^{m}:={ }_{\beta} \mathbf{A}^{n} \times \mathbf{A}^{m-n} .
$$

From (9), (10), and (11) we deduce that

$$
{ }_{\alpha} \mathbf{A}^{m} \stackrel{G}{\approx}{ }_{\gamma} \mathbf{A}^{m} \text {. }
$$

But $\gamma$ is linear because $\beta$ is. This and (12) complete the proof.

Lemma 3. For every affine algebraic group $G$ and every integer $r$ there exists a rational locally free linear action of $G$ on $\mathbf{A}^{s}$ for some $s>r$.

Proof. By [Bor91, Prop. 1.10], we may assume that $G$ is a closed subgroup of some $\mathrm{GL}_{n}$. As there is a closed embedding of $\mathrm{GL}_{n}$ in $\mathrm{GL}_{n+1}$, we may in addition assume that $n^{2}>r$. By Example 2 there is a rational locally free linear action $\alpha$ of $\mathrm{GL}_{n}$ on $\mathbf{A}^{n^{2}}$. Hence, $\left.\alpha\right|_{G}$ shares the requested properties.

Theorem 2. Every rational locally free action $\alpha$ of a special algebraic group $G$ on $\mathbf{A}^{m}$ is stably linearizable.

Proof. The same argument as in the proof of Theorem 1 shows that (7) holds. By Lemma 3 there is a rational locally free linear action $\gamma$ of $G$ on $\mathbf{A}^{s}$ for some $s \geqslant m$. Like for $\alpha$, for $\gamma$ we have

$$
{ }_{\gamma} \mathbf{A}^{s} \stackrel{G}{\approx}{ }_{\lambda} G \times\left({ }_{\gamma} \mathbf{A}^{s}{ }_{i}^{\prime} G\right)
$$

Let $d:=\operatorname{dim} G$. Since by [Che54] the underlying variety of $G$ is rational, we have

$$
G \approx \mathbf{A}^{d}
$$

From (7), (13), and (14) we then obtain

$$
\begin{aligned}
\mathbf{A}^{m} & \approx \mathbf{A}^{d} \times\left({ }_{\alpha} \mathbf{A}^{m} ; G\right), \\
\mathbf{A}^{s} & \approx \mathbf{A}^{d} \times\left({ }_{\gamma} \mathbf{A}^{s}{ }^{\prime} G\right) .
\end{aligned}
$$


In turn, (7), (13), and (15) imply

$$
\begin{aligned}
& { }_{\alpha} \mathbf{A}^{m} \times \mathbf{A}^{d} \stackrel{G}{\approx}{ }_{\lambda} G \times\left({ }_{\alpha} \mathbf{A}^{m}, G\right) \times \mathbf{A}^{d} \stackrel{G}{\approx}{ }_{\lambda} G \times \mathbf{A}^{m}, \\
& { }_{\gamma} \mathbf{A}^{s} \times \mathbf{A}^{d} \stackrel{G}{\approx}{ }_{\lambda} G \times\left({ }_{\gamma} \mathbf{A}^{s}{ }_{i}^{\prime} G\right) \times \mathbf{A}^{d} \stackrel{G}{\approx}{ }_{\lambda} G \times \mathbf{A}^{s} .
\end{aligned}
$$

Since $s \geqslant m$, we have $d+s-m \geqslant 0$ and from (16) we deduce

$$
\begin{aligned}
{ }_{\alpha} \mathbf{A}^{m} \times \mathbf{A}^{d+s-m} & ={ }_{\alpha} \mathbf{A}^{m} \times \mathbf{A}^{d} \times \mathbf{A}^{s-m} \\
& \stackrel{G}{\approx}{ }_{\lambda} G \times \mathbf{A}^{m} \times \mathbf{A}^{s-m} \\
& ={ }_{\lambda} G \times \mathbf{A}^{s} \\
& \stackrel{G}{\approx}{ }_{\gamma} \mathbf{A}^{s} \times \mathbf{A}^{d} .
\end{aligned}
$$

Since the action of $G$ on ${ }_{\gamma} \mathbf{A}^{s} \times \mathbf{A}^{d}$ is linear, (17) completes the proof.

The next theorem implies that "stably linearizable" in Theorem 2 cannot be replaced by "linearizable".

Theorem 3. For every connected semisimple algebraic group $G \neq\{e\}$, there exists a rational nonlinearizable locally free action of $G$ on $\mathbf{A}^{d}$ for $d=\operatorname{dim} G$.

Proof. Since (14) holds, there exists a rational locally free action $\alpha$ of $G$ on

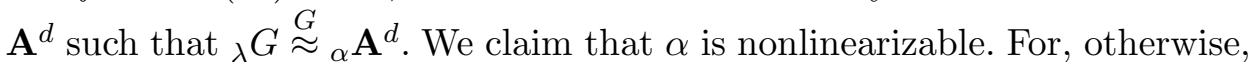
we would get a rational locally free linear (hence, regular) action of $G$ on $\mathbf{A}^{d}$. Since $d=\operatorname{dim} G$, one of its orbits is open in $\mathbf{A}^{d}$ and isomorphic to the underlying variety of $G$. Therefore, by Lemma 1 the center of $G$ is at least one-dimensional - a contradiction because $G$ is semisimple.

For tori we can get an additional information.

Lemma 4. Let $X$ be an irreducible variety endowed with a rational faithful action $\alpha$ of a torus $T$. Then

(i) $\alpha$ is locally free;

(ii) $\operatorname{dim} T \leqslant \operatorname{dim} X$;

(iii) $\operatorname{tr} \operatorname{deg}_{k} k(X)^{T}=\operatorname{dim} X-\operatorname{dim} T$.

Proof. By [Sum74, Cor.2 of Lemma 8] (see also [Bia66, Cor. 1 of Prop. 1]) there is an irreducible affine variety $Y$ endowed with a regular action of $T$ such that $X \stackrel{T}{\approx} Y$. Hence, we may (and shall) assume that $X$ is affine and $\alpha$ is regular. By [PV94, Theorem 1.5], we also may (and shall) assume that $X$ is a closed $T$-stable subset of a finite-dimensional algebraic $T$-module $V$ not contained in a proper $T$-submodule of $V$.

As $\alpha$ is faithful, the kernel of the action of $T$ on $V$ is trivial. As $T$ is a torus, $V$ is the direct sum of $T$-weight subspaces. Hence, if $U$ is the complement in $V$ to the union of these spaces, this kernel coincides with the $T$-stabilizer of every point of $U$. Thus, these stabilizers are trivial. But by the construction, $X \cap U$ is a nonempty open subset of $X$. This proves (i) that, in turn, entails (ii) and, by [PV94, Cor. in Sect. 2.3], also (iii).

Corollary 2 ([Dem70]). The dimension of every torus in $\mathcal{C}_{n}$ is at most $n$. 
Corollary 3. Every rational action of a torus on $\mathbf{A}^{n}$ is stably linearizable.

Proof. Since tori are special groups, this follows from Lemma 4(i) and Theorem 2.

Theorem 4. The following properties of a rational action $\alpha$ of a torus $T$ on $\mathbf{A}^{n}$ are equivalent:

(i) $\alpha$ is linearizable;

(ii) the invariant field $k\left({ }_{\alpha} \mathbf{A}^{n}\right)^{T}$ is pure over $k$.

Proof. Assume that (ii) holds. Let $T_{0}$ be the kernel of the action of $T$ on $X$. By Lemma 4, the induced action of $T / T_{0}$ on $\mathbf{A}^{n}$ is locally free. Hence, replacing $T$ by $T / T_{0}$, we may assume that the action of $T$ on $\mathbf{A}^{n}$ is locally free. Since $T$ is flattenable, in this case (ii) $\Rightarrow$ (i) follows from Theorem 1.

(i) $\Rightarrow$ (ii) is the corollary of the following more general statement.

Lemma 5. For any rational linear action $\alpha$ of a diagonalizable affine algebraic group $D$ on $\mathbf{A}^{n}$, the invariant field $k\left({ }_{\alpha} \mathbf{A}^{n}\right)^{D}$ is pure over $k$.

Proof of Lemma 5. By [Bor91, Prop. 8.2(d)], the image of $D$ under the homomorphism $D \rightarrow \mathrm{GL}_{n}$ determined by $\alpha$ is conjugate to a subgroup of $D_{n}$. Hence, we may (and shall) assume that $D$ is a closed subgroup of $D_{n}$. Since $\mathbf{A}^{n}$ with the natural action of $D_{n}$ is a flattening of $D_{n}$ (see Example 1), we have ${ }_{\alpha} \mathbf{A}^{n} \stackrel{D}{\approx}{ }_{\lambda} D_{n}$. Therefore,

$$
{ }_{\alpha} \mathbf{A}^{n}{ }_{i} D \approx D_{n} / D \text {. }
$$

But $D_{n} / D$ is a torus, see [Bor91, Props. 8.4 and 8.5], hence, a rational variety. The claim now follows from (18).

Corollary 4 ([Bia66, Cor. 2 of Prop. 1]).

(a) Every faithful rational action of a torus $T$ on $\mathbf{A}^{n}$ is linearizable in either of the following cases:

(i) $\operatorname{dim} T \geqslant n-2$;

(ii) $n \leqslant 3$.

(b) Every d-dimensional torus in $\mathcal{C}_{n}$ for $d=n-2, n-1, n$ is conjugate to a subgroup of $D_{n}$. In particular, every $n$-dimensional torus in $\mathcal{C}_{n}$ is conjugate to $D_{n}$.

Proof. (a) By Corollary 2, if $T \neq\{e\}$, then (ii) $\Rightarrow(\mathrm{i})$. Assume that (i) holds. Then $\operatorname{tr} \operatorname{deg}_{k} k\left(\mathbf{A}^{n}\right)^{T} \leqslant 2$ by Lemma 4(iii). As $k\left(\mathbf{A}^{n}\right)^{T}$ is unirational, it is then pure over $k$ by the Lüroth and Castelnuovo theorems; whence the claim by Theorem 4 .

Part (b) follows from (a).

By Corollaries 2 and 4 (b) all $n$-dimensional tori in $\mathcal{C}_{n}$ are maximal and conjugate and there are no maximal $(n-1)$ - and $(n-2)$-dimensional tori in $\mathcal{C}_{n}$. In dimension $n-3$ the situation is different:

Theorem 5. Let $n \geqslant 5$. Every $(n-3)$-dimensional connected affine algebraic group $G$ can be realized as an algebraic subgroup of $\mathcal{C}_{n}$ such that

(i) $k\left(\mathbf{A}^{n}\right)^{G}$ is not pure, but stably pure over $k$; 
(ii) the natural rational action of $G$ on $\mathbf{A}^{n}$ is locally free.

Proof. By [Sh-B04] there exists a nonrational threefold $X$ such that $\mathbf{A}^{2} \times$ $X \approx \mathbf{A}^{5}$. Then $\mathbf{A}^{n} \approx \mathbf{A}^{n-3} \times X$. This and (14) yield that there exists a rational locally free action $\gamma$ of $G$ on $\mathbf{A}^{n}$ such that ${ }_{\gamma} \mathbf{A}^{n} \stackrel{G}{\approx}{ }_{\lambda} G \times X$. Since $k\left({ }_{\lambda} G\right)^{G}=k$, by [Ros61 2 , Lemma 3], we have ${ }_{\gamma} \mathbf{A}^{n}{ }_{\prime}^{\prime} G \approx X$; whence the claim.

Corollary 5. Let $n \geqslant 5$. Then

(a) there is a rational locally free nonlinearizable action of an $(n-3)$ dimensional torus on $\mathbf{A}^{n}$;

(b) $\mathcal{C}_{n}$ contains an $(n-3)$-dimensional maximal torus.

Proof. Use the notation of Theorem 5 and its proof and let $G$ be a torus. Then $\gamma$ is nonlinearizable by Theorem 4. This proves (a). As the torus $G$ is not conjugate to a subgroup of $D_{n}$, Corollary 4(b) implies that it is maximal. This proves (b).

Corollary 6. Every $\mathcal{C}_{n}$ for $n \geqslant 5$ contains a nonlinearizable, but stably linearizable element of infinite order. ${ }^{1}$

Proof. For any subset $X$ of $\mathcal{C}_{n}$ denote by $\bar{X}$ the closure of $X$ in the Zariski topology of $\mathcal{C}_{n}$ (see Section 1). By Corollary $5(\mathrm{~b}), \mathcal{C}_{n}$ contains an $(n-3)$ dimensional maximal torus $T$. By [Bor91, Sect. III.8.8], there exists an element $g \in T$ such that $T=\bar{S}$ for $S:=\left\{g^{d} \mid d \in \mathbf{Z}\right\}$. Corollary 3 yields that $g$ is stably linearizable. Assume that $g$ is linearizable and let $h \in \mathcal{C}_{n}$ be an element such that $h g h^{-1} \in D_{n}$. Then $S \subset h^{-1} D_{n} h$. Since left and right translations of $\mathcal{C}_{n}$ are homeomorphisms and $\overline{D_{n}}=D_{n}$, we obtain

$$
T=\bar{S} \subset \overline{h^{-1} D_{n} h}=h^{-1} \overline{D_{n}} h=h^{-1} D_{n} h .
$$

This contradicts the maximality of $T$ because $h^{-1} D_{n} h$ is an $n$-dimensional torus.

The next statement yields a rectification of Corollaries 3 and 6 .

Theorem 6. Every torus $T$ in $\mathcal{C}_{m}$ is conjugate in $\mathcal{C}_{m+\operatorname{dim} T}$ to a subgroup of $D_{m+\operatorname{dim} T}$.

Proof. Let $\alpha$ be the natural rational action of $T$ on $\mathbf{A}^{m}$ and let $d:=\operatorname{dim} T$. By Lemma 4, $\alpha$ is locally free. By [Ros61 2 , Lemma 3], (7) and (14) we have

$$
\begin{aligned}
\left(\mathbf{A}^{d} \times{ }_{\alpha} \mathbf{A}^{m}\right)_{i}^{\prime} T & \approx \mathbf{A}^{d} \times\left({ }_{\alpha} \mathbf{A}^{m}{ }_{i} T\right), \\
{ }_{\alpha} \mathbf{A}^{m} & \stackrel{T}{\approx}{ }_{\lambda} T \times\left({ }_{\alpha} \mathbf{A}^{m}{ }_{i} T\right), \\
T & \approx \mathbf{A}^{d} .
\end{aligned}
$$

From (19) we deduce that $k\left({ }_{\alpha} \mathbf{A}^{m} \times \mathbf{A}^{d}\right)^{T}$ is pure over $k$. Since $T$ is flattenable, Theorem 1 then entails that $\mathbf{A}^{d} \times{ }_{\alpha} \mathbf{A}^{m} \stackrel{T}{\approx}{ }_{\gamma} \mathbf{A}^{m+d}$ for a rational linear action $\gamma$; whence the claim.

\footnotetext{
${ }^{1}$ In the original version of this paper (see arXiv:1110.2410v1-v3) the inequality $n \geqslant 5$ in Theorem 5 and Corollaries 5,6 , and 11 was replaced by $n \geqslant 6$ because I used the result of [BCSS85] in place of that of [Sh-B04], about which I was unaware.
} 


\section{Subgroups of the RATiOnAl DE Jonquières Groups}

Let $t_{1}, \ldots, t_{n}$ be a system of generators of $k\left(\mathbf{A}^{n}\right)$ over $k$,

$$
k\left(\mathbf{A}^{n}\right)=k\left(t_{1}, \ldots, t_{n}\right) .
$$

The elements $t_{1}, \ldots, t_{n}$ are algebraically independent over $k$ and determine the following flag of subfields of $k\left(\mathbf{A}^{n}\right)$ :

$$
K_{n} \subset K_{n-1} \subset \cdots \subset K_{0} \text {, where } K_{i}:= \begin{cases}k\left(t_{i+1}, t_{i+2}, \ldots, t_{n}\right) & \text { if } i \leqslant n-1, \\ k & \text { if } i=n .\end{cases}
$$

For any elements $f_{i} \in K_{i}$ and $\mu_{i} \in k^{\times}, i=1, \ldots, n$, put

$$
t_{i}^{\prime}:=\mu_{i} t_{i}+f_{i} \text { and } K_{i}^{\prime}:= \begin{cases}k\left(t_{i+1}^{\prime}, t_{i+2}^{\prime}, \ldots, t_{n}^{\prime}\right) & \text { if } i \leqslant n-1, \\ k & \text { if } i=n .\end{cases}
$$

It follows from (21) that there are elements $f_{i}^{\prime} \in K_{i}^{\prime}, i=1, \ldots, n$, such that

$$
t_{i}=\mu_{i}^{-1} t_{i}^{\prime}+f_{i}^{\prime} \text {. }
$$

Hence, $K_{i}^{\prime}=K_{i}$ for every $i$. In particular, $t_{1}^{\prime}, \ldots, t_{n}^{\prime}$ is an algebraically independent system of generators of $k\left(\mathbf{A}^{n}\right)$ over $k$, so there is an element $g \in \mathcal{C}_{n}$ such that

$$
g \cdot t_{i}=\mu_{i} t_{i}+f_{i} \quad \text { for every } \quad i=1, \ldots, n .
$$

The set $\mathcal{J}\left(t_{1}, \ldots, t_{n}\right)$ of all such elements $g$ is a subgroup of $\mathcal{C}_{n}$. It stabilizes the flag of subfields (20):

$$
g \cdot K_{i}=K_{i} \quad \text { for all } g \in \mathcal{J}\left(t_{1}, \ldots, t_{n}\right) \text { and } i=0, \ldots, n .
$$

If $s_{1}, \ldots, s_{n}$ is another system of generators of of $k\left(\mathbf{A}^{n}\right)$ over $k$, then the subgroups $\mathcal{J}\left(t_{1}, \ldots, t_{n}\right)$ and $\mathcal{J}\left(s_{1}, \ldots, s_{n}\right)$ are conjugate in $\mathcal{C}_{n}$.

Given an analogy of the construction of $\mathcal{J}\left(t_{1}, \ldots, t_{n}\right)$ with that of the de Jonquières subgroup of $\mathrm{Aut}_{k} k\left[t_{1}, \ldots, t_{n}\right]$, cf. [vdE00, p. 85], we call $\mathcal{J}\left(t_{1}, \ldots\right.$ $\left.\ldots, t_{n}\right)$ the rational de Jonquières subgroup of $\mathcal{C}_{n}$ with respect to $t_{1}, \ldots, t_{n}$.

Example 6. By the Lie-Kolchin theorem every closed connected solvable subgroup $G$ of $\mathrm{GL}_{n}$ is conjugate in $\mathrm{GL}_{n}$ to a subgroup of $\mathcal{J}\left(x_{1}, \ldots, x_{n}\right)$. Hence, $G$ lies in $\mathcal{J}\left(t_{1}, \ldots, t_{n}\right)$ where $t_{1}, \ldots, t_{n}$ are the homogeneous linear forms in $x_{1}, \ldots, x_{n}$.

In the notation of $(22)$, for every $i=1, \ldots, n$, we have the following maps:

$$
\begin{aligned}
\chi_{i}: \mathcal{J}\left(t_{1}, \ldots, t_{n}\right) \rightarrow k^{\times}, & g \mapsto \mu_{i}, \\
\varphi_{i}: \mathcal{J}\left(t_{1}, \ldots, t_{n}\right) \rightarrow K_{i}, & g \mapsto f_{i} .
\end{aligned}
$$

Lemma 6. For every $i=1, \ldots, n$,

(a) $\chi_{i}$ is a homomorphism of groups;

(b) for all $g_{1}, g_{2} \in \mathcal{J}\left(t_{1}, \ldots, t_{n}\right)$,

$$
\varphi_{i}\left(g_{1} g_{2}\right)=\chi_{i}\left(g_{2}\right) \varphi_{i}\left(g_{1}\right)+g_{1} \cdot\left(\varphi_{i}\left(g_{2}\right)\right) ;
$$

(c) if $G$ is an algebraic subgroup of $\mathcal{C}_{n}$ contained in $\mathcal{J}\left(t_{1}, \ldots, t_{n}\right)$, then $\left.\chi_{i}\right|_{G}$ is a regular function on $G$ and there is a rational function $F_{i} \in$ $k\left(G \times \mathbf{A}^{n}\right)$ such that $F_{i}(g)=\varphi_{i}(g)$ for all $g \in G$;

(d) the order of every element $g \in \bigcap_{i=1}^{n} \operatorname{ker} \chi_{i}, g \neq e$, is infinite. 
Proof. Let $g_{1}, g_{2} \in \mathcal{J}\left(t_{1}, \ldots, t_{n}\right)$. Then (22) and (24) yield

$$
\begin{aligned}
\chi_{i}\left(g_{1} g_{2}\right) t_{i}+\varphi_{i}\left(g_{1} g_{2}\right) & =g_{1} g_{2} \cdot t_{i}=g_{1} \cdot\left(g_{2} \cdot t_{i}\right) \\
& =g_{1} \cdot\left(\chi_{i}\left(g_{2}\right) t_{i}+\varphi_{i}\left(g_{2}\right)\right) \\
& =\chi_{i}\left(g_{2}\right)\left(\chi_{i}\left(g_{1}\right) t_{i}+\varphi_{i}\left(g_{1}\right)\right)+g_{1} \cdot\left(\varphi_{i}\left(g_{2}\right)\right) .
\end{aligned}
$$

As the image of $\varphi_{i}$ lies in the $\mathcal{J}\left(t_{1}, \ldots, t_{n}\right)$-stable field $K_{i},(26)$ and algebraic independence of $t_{1}, \ldots, t_{n}$ over $k$ yield that $(25)$ and $\chi_{i}\left(g_{1} g_{2}\right)=\chi_{i}\left(g_{1}\right) \chi_{i}\left(g_{2}\right)$ hold. This proves (a) and (b).

(c) Let $\alpha: G \times \mathbf{A}^{n} \rightarrow \mathbf{A}^{n}$ be the natural rational action of $G$ on $\mathbf{A}^{n}$ and let $\beta: G \times \mathbf{A}^{n} \rightarrow G \times \mathbf{A}^{n},(g, a) \mapsto\left(g^{-1}, a\right)$. Put $S_{i}:=\beta^{*}\left(\alpha^{*}\left(t_{i}\right)\right) \in k\left(G \times \mathbf{A}^{n}\right)$. Then $S_{i}(g, a)=t_{i}(\alpha(\beta(g, a)))=t_{i}\left(\alpha\left(g^{-1}, a\right)\right)=t_{i}\left(g^{-1} \cdot a\right)=\left(g \cdot t_{i}\right)(a)$ for every $(g, a)$ in the domain of definition. Hence, $S_{i}(g)=\chi_{i}(g) t_{i}+\varphi_{i}(g)$ for every $g \in G$. Given that $S_{i} \in k\left(G \times \mathbf{A}^{n}\right)=k(G)\left(t_{1}, \ldots, t_{n}\right)$ and $\varphi_{i}(g) \in K_{i}$, this implies (c).

(d) As $g \neq e$ and $\chi_{i}(g)=1$ for every $i,(22)$ and (24) entail that there is $j$ such that $\varphi_{j}(g) \neq 0$. Let $d$ be the largest $j$ with this property. Then $g \cdot f=f$ for every $f \in K_{d}$. As $g \cdot t_{d}=t_{d}+\varphi_{d}(g)$ and $\varphi_{d}(g) \in K_{d}$, this yields

$$
g^{s} \cdot t_{d}=t_{d}+s \varphi_{d}(g) \quad \text { for every } s \in \mathbf{Z} \text {. }
$$

Since $\varphi_{d}(g) \neq 0$ and char $k=0,(27)$ implies that $g^{s} \neq e$ for every $s \neq 0$. This proves $(\mathrm{d})$.

Theorem 7. Let $G$ be an affine algebraic subgroup of $\mathcal{J}\left(t_{1}, \ldots, t_{n}\right)$. Then $G$ is solvable and $G / G^{0}$ is Abelian.

Proof. First, consider the case where $G$ is finite; we then have to prove that $G$ is Abelian. Consider the homomorphism

$$
\delta: \mathcal{J}\left(t_{1}, \ldots, t_{n}\right) \rightarrow D_{n}, \quad g \mapsto \operatorname{diag}\left(\chi_{1}(g), \ldots, \chi_{n}(g)\right) .
$$

Since $\operatorname{ker} \delta=\bigcap_{i=1}^{n} \operatorname{ker} \chi_{i}$ and $G$ has no elements of infinite order, Lemma $6(\mathrm{~d})$ implies that $G \cap \operatorname{ker} \delta=\{e\}$. Therefore, $\delta$ embeds $G$ into the Abelian group $D_{n}$; whence, the claim.

Now consider the general case. By [BS64, Lemma 5.11], there is a finite subgroup $H$ of $G$ that intersects every connected component of $G$. Hence, the restriction to $H$ of the canonical homomorphism $G \rightarrow G / G^{0}$ is a surjective homomorphism $H \rightarrow G / G^{0}$. According to what we have already proved, $H$ is Abelian. This shows that $G / G^{0}$ is Abelian. By [Hal59, Theorem 9.2.5], the problem is then reduced to proving that $G^{0}$ is solvable.

Since $\operatorname{char} k=0$, there exists a Levi subgroup $L$ in $G^{0}$, see [Bor91, 11.22]. It is a connected reductive group and we have to show that $L$ is a torus, i.e., that the derived subgroup $L^{\prime}$ of $L$ is trivial. Arguing on the contrary, assume that $L^{\prime} \neq\{e\}$. Then $L^{\prime}$ contains an element $g \neq e$ of finite order. Indeed, $L^{\prime}$ contains a torus $\neq\{e\}$ (see [Bor91, Cor. 2 in Sect. IV.13.17 and Theorem 12.1(b)]), but every torus $\neq\{e\}$ has a nontrivial torsion (see [Bor91, Prop. 8.9(d)]). On the other hand, $L^{\prime} \subseteq \bigcap_{i=1}^{n}$ ker $\chi_{i}$ as every homomorphism $L \rightarrow k^{\times}$contains $L^{\prime}$ in its kernel. By Lemma 6(d) this entails that the order of $g$ is infinite. This contradiction completes the proof. 
Corollary 7. Every finite subgroup of $\mathcal{J}\left(t_{1}, \ldots, t_{n}\right)$ is Abelian.

Theorem 8. Let $G$ be a reductive algebraic subgroup of $\mathcal{J}\left(t_{1}, \ldots, t_{n}\right)$. Then $G$ is a diagonalizable group.

Proof. By Theorem 7 the reductive group $G^{0}$ is solvable. Hence, $G^{0}$ is a torus. Let $H$ be the subgroup of $G$ from the proof of Theorem 7. It acts on $G^{0}$ by conjugation because $G^{0}$ is normal in $G$. The fixed point set $F$ of this action is a closed subgroup of $G^{0}$. Assume that $F \neq G^{0}$. Then, since the torsion subgroup of $G^{0}$ is dense in $G^{0}$ (see [Bor91, Cor. III.8.9]), there exists an element $g \in G^{0} \backslash F$ whose order is finite. Let $S$ be the subgroup of $G^{0}$ generated by the set $\left\{h g h^{-1} \mid h \in H\right\}$. Since $G^{0}$ is Abelian and the orders of $g$ and $H$ are finite, $S$ is finite as well. Since $S$ is stable with respect to the action of $H$ on $G^{0}$ by conjugation, this implies that the subgroup generated by $S$ and $H$ is finite, too. Corollary 7 then yields that this subgroup is Abelian. Hence, $g \in F-$ a contradiction. Therefore, $F=G^{0}$, i.e., $G^{0}$ and $H$ commute. Since the Abelian groups $H$ and $G^{0}$ generate $G$, this implies that $G$ is Abelain. The claim then follows by [Bor91, Prop. III.8.4(4) and Cor. III.4.4(1)].

\section{Affine subspaces as CROSS-Sections}

By [Ros56, Theorem 10], for every rational action of a connected solvable algebraic group there exists a rational cross-section. The next theorem refines this for some rational actions on $\mathbf{A}^{n}$ by showing that there exist cross-sections that are affine subspaces of $\mathbf{A}^{n}$.

Theorem 9. Let $G \neq\{e\}$ be a unipotent affine algebraic subgroup of the group $\mathcal{J}\left(x_{1}, \ldots, x_{n}\right)$ and let $\alpha$ be the corresponding rational action of $G$ on $\mathbf{A}^{n}$. Then there exist a sequence $1 \leqslant i_{1}<\cdots<i_{m} \leqslant n$ of natural numbers and a sequence $\Theta_{1}, \ldots, \Theta_{m}$ of nonempty open subsets of $k$ such that for every $\left(c_{1}, \ldots, c_{m}\right) \in \Theta_{1} \times \cdots \times \Theta_{m}$ the affine subspace of $\mathbf{A}^{n}$ defined by the equations (see (3)):

$$
x_{i_{1}}=c_{1}, \ldots, x_{i_{m}}=c_{m}
$$

is a rational cross-section for $\alpha$.

For the proof of Theorem 9 we need the following

Lemma 7. Let $K$ be a field of characteristic 0 and let $f(x)$ be a rational function in a variable $x$ with the coefficients in $K$. Let $K^{\prime}$ be a subfield of K. If

$$
f\left(a_{1}+a_{2}\right)=f\left(a_{1}\right)+f\left(a_{2}\right)
$$

whenever $f$ is defined at $a_{1}, a_{2}$ and $a_{1}+a_{2} \in K^{\prime}$, then there is an element $c \in K$ such that $f(x)=c x$.

Proof of Lemma 7. We may (and shall) assume that $f \neq 0$. Let $\bar{K}$ be an algebraic closure of $K$. First, we claim that (28) holds whenever $f$ is defined at $a, b$ and $a+b \in \bar{K}$. Indeed, by (28) the rational function $F\left(x_{1}, x_{2}\right):=$ $f\left(x_{1}\right)+f\left(x_{2}\right)-f\left(x_{1}+x_{2}\right)$ (see $\left.(3)\right)$ vanishes at every point of $\mathbf{A}^{2}\left(K^{\prime}\right)$ where it is defined. Since $\mathbf{A}^{2}\left(K^{\prime}\right)$ is Zariski dense in $\mathbf{A}^{2}$, this yields $F=0$; whence the claim. 
Thus, $f$ is a rational partially defined endomorphism of the algebraic group $\bar{K}^{+}$. But by [Wei55] (cf. also [Mer80, Sect. 11.1.1]) every rational partially defined homomorphism of algebraic groups is, in fact, an everywhere defined algebraic homomorphism. This entails that $f(x) \in K[x]$. Since $f$ has only finitely many roots, $\operatorname{ker} f$ is finite. Therefore, $f\left(\bar{K}^{+}\right)$is a onedimensional closed subgroup of $\bar{K}^{+}$; whence $f\left(\bar{K}^{+}\right)=\bar{K}^{+}$. On the other hand, since char $K=0$, there are no nonzero elements of finite order in $\bar{K}^{+}$. Hence, ker $f=\{0\}$. Thus, $f$ is an isomorphism; whence the claim.

Proof of Theorem 9. We shall use the notation of (20), (24) with

$$
t_{1}=x_{1}, \ldots, t_{n}=x_{n} .
$$

Since char $k=0, G$ is connected. As $G$ is a nontrivial unipotent group, it contains a one-dimensional normal subgroup $U$ isomorphic to $k^{+}$. We identify $U$ with $k^{+}$by an isomorphism $U \rightarrow k^{+}$. Since $G$ is unipotent, there are no nontrivial algebraic homomorphisms $G \rightarrow k^{\times}$, therefore, by Lemma 6 there are rational functions $F_{i} \in k\left(G \times \mathbf{A}^{n}\right)$ such that

$$
\begin{aligned}
& g \cdot x_{i}=x_{i}+F_{i}(g), \quad \text { for every } g \in G \text { and } i . \\
& F_{i}(g) \in K_{i},
\end{aligned}
$$

Since $U \neq\{e\},(29)$ entails that $F_{j}(u) \neq 0$ for some $u \in U$ and $j$. Let $d$ be the largest $j$ appearing in this fashion. Then (29) and (20) yield

$$
K_{d}^{U}=K_{d} .
$$

In turn, from (30) and (25) we infer that

$$
F_{d}\left(u_{1}+u_{2}\right)=F_{d}\left(u_{1}\right)+F_{d}\left(u_{2}\right) \quad \text { for all } u_{1}, u_{2} \in U \text {. }
$$

By Lemma 7, this implies that there is a nonzero element $s \in K_{d}$ such that

$$
F_{d}(u)=u s \quad \text { for every } u \in U .
$$

Thus, by (29) and (31),

$$
u \cdot x_{d}=x_{d}+u s, \quad \text { for every } u \in U .
$$

By [Ros56, Theorem 1], there exists a nonempty open subset $\mathbf{A}_{0}^{n}$ of $\mathbf{A}^{n}$ and its embedding in an irreducible variety $Y$,

$$
Y \hookleftarrow \mathbf{A}_{0}^{n} \subseteq \mathbf{A}^{n},
$$

such that the rational action of $U$ on $Y$ determined by $\left.\alpha\right|_{U}$ and by this embedding is regular. We identify $\mathbf{A}_{0}^{n}$ with the image of this embedding. By [Ros56, Theorem 2], shrinking $Y$ if necessary, we may (and shall) assume that there exists a geometric quotient of $Y$ by this action of $U$,

$$
\pi_{U, Y}: Y \rightarrow Y / U
$$

Then $\left.\pi_{U, Y}\right|_{\mathbf{A}_{0}^{n}}$ is the restriction to $\mathbf{A}_{0}^{n}$ of a rational quotient for $\left.\alpha\right|_{U}$,

$$
\pi_{U, \mathbf{A}^{n}}: \mathbf{A}^{n} \rightarrow Y / U=: \mathbf{A}^{n}{ }^{\prime} U .
$$


Let $H:=G / U$. Then $\alpha$ induces a rational action $\beta$ of $H$ on $Y / U$. Consider a rational quotient for $\beta$,

$$
\pi_{H, Y / U}: Y / U \rightarrow(Y / U)_{i}^{\prime} H .
$$

Then the composition

$$
\pi_{G, \mathbf{A}^{n}}:=\pi_{H, Y / U} \circ \pi_{U, \mathbf{A}^{n}}
$$

is a rational quotient for $\alpha$,

$$
\pi_{G, \mathbf{A}^{n}}: \mathbf{A}^{n} \rightarrow(Y / U)_{\prime}^{\prime} H=: \mathbf{A}^{n} i^{\prime} G
$$

Shrinking $\mathbf{A}_{0}^{n}$ and $Y$ if necessary, we may (and shall) assume that

(i) $\pi_{H, Y / U}$ is a morphism;

(ii) $\left.s\right|_{\mathbf{A}_{0}^{n}}$ is regular and vanishes nowhere.

To sum up, we have the following commutative diagram:

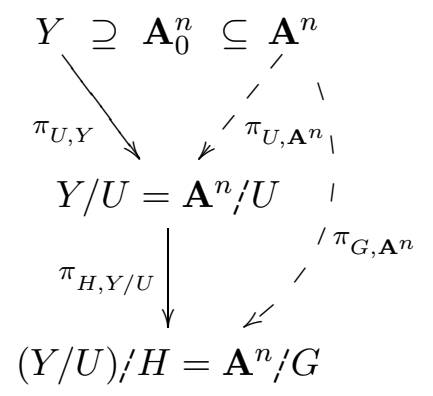

For every element $c \in k$, denote by $L_{c}$ the hyperplane in $\mathbf{A}^{n}$ defined by the equation $x_{d}=c$. The set

$$
\Omega:=\left\{c \in k \mid L_{c} \cap \mathbf{A}_{0}^{n} \neq \varnothing\right\}
$$

is nonempty and open in $k$. Take an element $c \in \Omega$ and a point $a \in \mathbf{A}_{0}^{n}$. By property (ii) above, $s$ is regular and does not vanish at $a$. Consider the $U$-orbit of $a$ in $Y$. Formula (31) shows that there is a unique $u_{0} \in U$ such that the value of $x_{d} \in k(Y)$ at $u_{0} \cdot a$ is $c$, namely,

$$
u_{0}=\frac{x_{d}-c}{s}(a) \text {. }
$$

This means that every $U$-orbit in $Y$ intersects $L_{c} \cap \mathbf{A}_{0}^{n}$ at most at one point, i.e., $\left.\pi_{U, Y}\right|_{L_{c} \cap \mathbf{A}_{0}^{n}}$ is injective. Since $\operatorname{dim} L_{c} \cap \mathbf{A}_{0}^{n}=\operatorname{dim} Y / U$ and $\operatorname{char} k=0$, this implies that $\left.\pi_{U, Y}\right|_{L_{c} \cap \mathbf{A}_{0}^{n}}: L_{c} \cap \mathbf{A}_{0}^{n} \rightarrow Y / U$ is a birational isomorphism. Hence, $L_{c}$ intersects the domain of definition of $\pi_{U, \mathbf{A}^{n}}$ and

$$
\left.\pi_{U, \mathbf{A}^{n}}\right|_{L_{c}}: L_{c} \rightarrow Y / U=\mathbf{A}^{n}{ }^{\prime} U,
$$

is a birational isomorphism. This means that $L_{c}$ is a rational cross-section for $\left.\alpha\right|_{U}$. In particular, this implies that shrinking $\mathbf{A}_{0}^{n}$ if necessary, we may (and shall) assume that

(iii) for every point of $\mathbf{A}_{0}^{n}$, its $U$-orbit in $Y$ intersects $L_{c}$. 
Now we argue by induction on $\operatorname{dim} G$. If $\operatorname{dim} G=1$, then $G=U$ and the claim is proved since every $L_{c}$ for $c \in \Omega$ is a rational cross-section for $\alpha$ (so in this case $s=1, i_{1}=d$ and $\Theta_{1}=\Omega$ ).

Now assume that $\operatorname{dim} G>1$. The action $\beta$ and the birational isomorphism (35) determine a rational action $\gamma$ of $H$ on $L_{c}$ such that (35) becomes an $H$-equivariant birational isomorphism. From (33) we then deduce that

$$
\pi_{G,\left.\mathbf{A}^{n}\right|_{L_{c}}: L_{c} \rightarrow \mathbf{A}^{n}{ }^{\prime} G}
$$

is a rational quotient for $\gamma$. We identify $L_{c}$ with $\mathbf{A}^{n-1}$ by means of the isomorphism $\left(a_{1}, \ldots, a_{d-1}, c, a_{d+1}, \ldots, a_{n}\right) \mapsto\left(a_{1}, \ldots, a_{d-1}, a_{d+1}, \ldots, a_{n}\right)$ and, for every function $f \in k\left(\mathbf{A}^{n}\right)$ whose domain of definition intersects $L_{c}$, put

$$
\bar{f}:=\left.f\right|_{L_{c}} \in k\left(L_{c}\right) .
$$

Then $\overline{x_{1}}, \ldots, \overline{x_{d-1}}, \overline{x_{d+1}}, \ldots, \overline{x_{n}}$ are the standard coordinate functions on $L_{c}$.

We claim that the image of $H$ in $\operatorname{Aut}_{k} k\left(L_{c}\right)=\mathcal{C}_{n-1}$ determined by the action $\gamma$ is contained in $\mathcal{J}\left(\overline{x_{1}}, \ldots, \overline{x_{d-1}}, \overline{x_{d+1}}, \ldots, \overline{x_{n}}\right)$. If this is proved, then, by the inductive assumption, there exist a nonempty set of indices $i_{1}, \ldots, i_{r}$ and a nonempty open subsets $\Theta_{1}, \ldots, \Theta_{r}$ of $k$ such that for every $\left(c_{1}, \ldots, c_{r}\right) \in \Theta_{1} \times \cdots \times \Theta_{r}$ the affine subspace $S$ of $L_{c}$ defined by the equations

$$
\overline{x_{i_{1}}}=c_{1}, \ldots, \overline{x_{i_{r}}}=c_{r},
$$

is a rational cross-section for $\gamma$, i.e.,

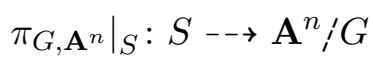

is a birational isomorphism. As $S$ is an affine subspace in $\mathbf{A}^{n}$ defined by the equations $x_{d}=c, x_{i_{1}}=c_{1}, \ldots, x_{i_{r}}=c_{r}$, this will complete the proof.

It remains to prove the claim. To this end, consider in $k\left(\mathbf{A}^{n}\right)$ the subfield $k\left(\mathbf{A}^{n}\right)^{U}$ of $U$-invariants elements with respect to $\left.\alpha\right|_{U}$. Since $L_{c}$ is a rational cross-section of $\pi_{U, \mathbf{A}^{n}}$, the map

$$
k\left(\mathbf{A}^{n}\right)^{U} \rightarrow k\left(L_{c}\right), \quad f \mapsto \bar{f},
$$

is a well-defined $k$-isomorphism of fields that is $H$-equivariant with respect to the actions of $H$ on $k\left(\mathbf{A}^{n}\right)^{U}$ and $k\left(L_{c}\right)$ determined resp. by $\alpha$ and $\gamma$. Let

$$
k\left(L_{c}\right) \rightarrow k\left(\mathbf{A}^{n}\right)^{U}, \quad t \mapsto \widehat{t},
$$

be the inverse isomorphism. Below we will consider $\beta$ and $\gamma$ as the actions of $G$ with the kernel $U$.

Take a point $a \in \mathbf{A}_{0}^{n}$. By the above discussion and property (iii), the $U$ orbit of $a$ in $Y$ intersects $L_{c}$ at a single point $u_{0} \cdot a$ where $u_{0}$ is given by (34). As $\widehat{\overline{x_{i}}} \in k\left(\mathbf{A}^{n}\right)^{U}$, this yields

$$
\widehat{\overline{x_{i}}}(a)=\widehat{\overline{x_{i}}}\left(u_{0} \cdot a\right)=\overline{x_{i}}\left(u_{0} \cdot a\right)=x_{i}\left(u_{0} \cdot a\right)=\left(\left(-u_{0}\right) \cdot x_{i}\right)(a) .
$$

Let $z, y_{1}, \ldots, y_{n-1}$ be the variables over $k$. It follows from (29), (34) and (36) that there are $d-1$ rational functions

$$
R_{j}\left(z, y_{j}, y_{j+1}, \ldots, y_{n-1}\right) \in k\left(z, y_{j}, y_{j+1}, \ldots, y_{n-1}\right), \quad j=1, \ldots, d-1,
$$


such that

$$
\widehat{\overline{x_{i}}}= \begin{cases}x_{i}+R_{i}\left(\frac{c-x_{d}}{s}, x_{i+1}, \ldots, x_{n}\right) & \text { if } i \leqslant d-1, \\ x_{i} & \text { if } i \geqslant d+1 .\end{cases}
$$

In turn, from (37), (29), (20) and (23) we infer that

$$
g \cdot \widehat{\overline{x_{i}}}-x_{i} \in k\left(x_{i+1}, \ldots, x_{d-1}, x_{d}, x_{d+1}, \ldots, x_{n}\right) \text { for all } g \in G \text { and } i \text {; }
$$

whence,

$$
g \cdot \overline{x_{i}}-\overline{x_{i}} \in k\left(\overline{x_{i+1}}, \ldots, \overline{x_{d-1}}, \overline{x_{d}}, \overline{x_{d+1}}, \ldots, \overline{x_{n}}\right) \text { for all } g \in G \text { and } i \text {. }
$$

The claim now follows from (38) because $\overline{x_{d}}=c \in k$.

Corollary 8. For every unipotent algebraic subgroup $G$ of $\mathrm{GL}_{n}$, there exists an affine subspace $L$ of $\mathbf{A}^{n}$ such that $L$ is a rational cross-section for the natural action of $G$ on $\mathbf{A}^{n}$.

Proof. There exists an element $g \in \mathrm{GL}_{n}$ such that $g G g^{-1} \subset \mathcal{J}\left(x_{1}, \ldots, x_{n}\right)$ (see Example 6). By Theorem 9 there exists an affine subspace $S$ of $\mathbf{A}^{n}$ that is a rational cross-section for the natural action of $g G g^{-1}$ on $\mathbf{A}^{n}$. Then the affine subspace $g^{-1}(S)$ is a rational cross-section for the natural action of $G$ on $\mathbf{A}^{n}$.

Here is the application of Corollary 8. Let $G$ be a connected affine algebraic group and let $\mathfrak{g}$ be the Lie algebra of $G$. Joseph put forward the following

Conjecture A ([Jos11, Sect. 7.11]). For the coadjoint action of $G$ on $\mathfrak{g}^{*}$, there exists an affine subspace $L$ of $\mathfrak{g}^{*}$ such that $k\left(\mathfrak{g}^{*}\right)^{G} \rightarrow k(L),\left.f \mapsto f\right|_{L}$, is a well-defined isomorphism of fields.

Joseph calls such $L$ a rational slice.

According to the Levi decomposition, $\mathfrak{g}$ is a semidirect product of a reductive Lie algebra $\mathfrak{r}$ and the unipotent radical $\mathfrak{u}$,

$$
\mathfrak{g}=\mathfrak{r} \ltimes \mathfrak{u} .
$$

Corollary 9. If (39) is a direct product, $\mathfrak{g}=\mathfrak{r} \times \mathfrak{u}$, then Conjecture $A$ is true.

Proof. Let $R$ and $U$ be the closed connected subgroups of $G$ whose Lie algebras are resp. $\mathfrak{r}$ and $\mathfrak{u}$. Assume that $\mathfrak{g}=\mathfrak{r} \times \mathfrak{u}$. In this case, if $L_{\mathfrak{r}}$ and $L_{\mathfrak{u}}$ are the rational slices for the coadjoint actions of resp. $R$ and $U$, then $L_{\mathfrak{r}} \times L_{\mathfrak{u}}$ is a rational slice for the coadjoint action of $G$. The existence of $L_{\mathfrak{r}}$ is proved in [Kos63] and the existence of $L_{\mathfrak{u}}$ is ensured by Corollary 8 .

Remark 1. Another application is that Theorem 9 yields the results of $[\mathrm{Pu}$, Part II, Chap. I, §7].

The rational de Jonquières subgroup $\mathcal{J}\left(t_{1}, \ldots, t_{n}\right)$ lies in another interesting subgroup of $\mathcal{C}_{n}$. Namely, as for $\mathcal{J}\left(t_{1}, \ldots, t_{n}\right)$, one checks that, for every $f_{i} \in K_{i}$ and $\mu_{i} \in K_{i}^{\times}$, there exists an element $g \in \mathcal{C}_{n}$ for which (22) holds 
and that the set $\widehat{\mathcal{J}}\left(t_{1}, \ldots, t_{n}\right)$ of all such elements $g$ is a subgroup of $\mathcal{C}_{n}$. The flag of subfields (20) is stable with respect to $\widehat{\mathcal{J}}\left(t_{1}, \ldots, t_{n}\right)$ :

$$
g \cdot K_{i}=K_{i} \quad \text { for all } g \in \widehat{\mathcal{J}}\left(t_{1}, \ldots, t_{n}\right) \text { and } i .
$$

If $s_{1}, \ldots, s_{n}$ is another system of generators of of $k\left(\mathbf{A}^{n}\right)$ over $k$, then the subgroups $\widehat{\mathcal{J}}\left(t_{1}, \ldots, t_{n}\right)$ and $\widehat{\mathcal{J}}\left(s_{1}, \ldots, s_{n}\right)$ are conjugate in $\mathcal{C}_{n}$.

The following fact is known; it provides an information on tori in $\widehat{\mathcal{J}}\left(t_{1}, \ldots\right.$ $\ldots, t_{n}$ ) (see Corollary 10 below).

Theorem 10. For every (not necessarily algebraic) subgroup $G$ of the group $\widehat{\mathcal{J}}\left(t_{1}, \ldots, t_{n}\right)$, the invariant field $k\left(\mathbf{A}^{n}\right)^{G}$ is pure over $k$.

Proof. We shall sketch a proof since our argument provides a bit more information (equality (43)) than that of [Miy71] and [KV89]. The key ingredient is the following Miyata's lemma:

Lemma 8 ([Miy71, Lemma], cf. [KV89, Lemme 1.1], [AHK00, Sect. 3]). Let $F$ be a field, let $z$ be a variable over $F$, and let $H$ be a group that acts on $F[z]$ by ring automorphisms leaving $F$ stable ${ }^{2}$. Then the subfield of $F(z)$ generated by $F(z)^{H}$ over $F$ is, in fact, generated by a single element $x \in F[z]^{H}$ :

$$
F\left(F(z)^{H}\right)=F(x) .
$$

Turning to the proof of Theorem 10, we first show that, in the notation of Lemma 8,

$$
F(z)^{H}=F^{H}(x)
$$

Indeed, $F(x)^{H} \subseteq F(z)^{H}$ since $F(x) \subseteq F(z)$. On the other hand, (41) entails that $F(z)^{H} \subseteq \bar{F}(x)^{H}$. Hence, $F(z)^{\bar{H}}=F(x)^{H}$. Therefore, (42) would be proved if the equality

$$
F(x)^{H}=F^{H}(x)
$$

is established. To prove (43), consider two cases: (a) $x \in F$, (b) $x \notin F$. If (a) holds, then $F(x)=F$, hence, $F(x)^{H}=F^{H}$. On the other hand, (a) and $x \in F[z]^{H}$ yield that $x \in F^{H}$, hence, $F^{H}(x)=F^{H}$. This proves (43) if (a) holds. Now assume that (b) holds. Then $x$ is transcendental over $F$ by [vdW67, $\S 73$, Theorem]. Consider an element $f \in F(x)^{H}$. It can be written as $f=p / q$ where

$$
p=\sum_{i=0}^{s} a_{i} x^{i}, \quad q=\sum_{j=0}^{r} b_{j} x^{j}, \quad a_{i}, b_{j} \in F, \quad a_{s} b_{r} \neq 0,
$$

and $p$ and $q$ are relatively prime polynomials in $x$ with the coefficients in $F$. Since $F[x]$ is a factorial ring, the relative primeness of $p$ and $q$ and $H$ invariance of $f$ imply that there is a map $\gamma: H \rightarrow F^{*}$ (in fact, a 1-cocycle) such that

$$
h \cdot p=\gamma(h) p, \quad h \cdot q=\gamma(h) q \quad \text { for every } h \in H .
$$

Since $x$ is $H$-invariant, (44) and (45) yield

$$
h \cdot a_{i}=\gamma(h) a_{i}, \quad h \cdot b_{j}=\gamma(h) b_{j} \quad \text { for all } h \in H \text { and } i, j .
$$

\footnotetext{
${ }^{2}$ It is not assumed that $F$ is pointwise fixed.
} 
From (46) we infer that $f=a_{s}^{-1} p / a_{s}^{-1} q \in F^{H}(x)$. Thus, $F(x)^{H} \subseteq F^{H}(x)$. Since $x$ is $H$-invariant, the inverse inclusion is clear. This proves (43). Thus, (42) holds and, moreover, either $x \in F^{H}$ or $x$ is transcendental over $F$.

Now let $G$ be a subgroup of $\widehat{\mathcal{J}}\left(t_{1}, \ldots, t_{n}\right)$. We have $K_{i-1}=K_{i}\left(t_{i}\right)$ and $t_{i}$ is transcendental over $K_{i}$ for every $i=1, \ldots, n$. By (40) and the definition of $\widehat{\mathcal{J}}\left(t_{1}, \ldots, t_{n}\right)$ the action of $G$ on $K_{i-1}$ satisfies the conditions of Lemma 8 (with $F=K_{i}, z=t_{i}, H=G$ ). Hence, as is proved above, there is an element $z_{i} \in K_{i}\left[t_{i}\right]^{G}$ such that $K_{i-1}^{G}=K_{i}\left(t_{i}\right)^{G}=K_{i}^{G}\left(z_{i}\right)$ and either $z_{i} \in K_{i}^{G}$ or $z_{i}$ is transcendental over $K_{i}$. Respectively, either $K_{i-1}^{G}=K_{i}^{G}$ or $K_{i-1}^{G}$ is pure over $K_{i}^{G}$ of transcendental degree 1. Since

$$
k=K_{n}^{G} \subseteq K_{n-1}^{G} \subseteq \cdots \subseteq K_{1}^{G} \subseteq K_{0}^{G}=k\left(\mathbf{A}^{n}\right)^{G},
$$

this completes the proof.

Corollary 10. Every torus in $\widehat{\mathcal{J}}\left(t_{1}, \ldots, t_{n}\right)$ is conjugate in $\mathcal{C}_{n}$ to a subgroup of $D_{n}$.

Proof. This follows from Theorems 4 and 10.

Corollary 11. Let $n \geqslant 5$. Every $(n-3)$-dimensional connected affine algebraic group can be realized as an algebraic subgroup of $\mathcal{C}_{n}$ such that

(i) $G$ is not conjugate to a subgroup of $\widehat{\mathcal{J}}\left(t_{1}, \ldots, t_{n}\right)$;

(ii) the natural rational action of $G$ on $\mathbf{A}^{n}$ is locally free.

Proof. This follows from Theorems 5 and 10.

Remark 2. The assumption that $k$ is algebraically closed is not used in the proof of Theorem 10.

Remark 3. In [Miy71], Lemma 8 is used for proving that $k\left(\mathbf{A}^{n}\right)^{G}$ is pure over $k$ if $G$ is a subgroup of $\mathrm{GL}_{n} \cap \mathcal{J}\left(x_{1}, \ldots, x_{n}\right)$. Note that in this case, if $G$ is finite, then purity of $k\left(\mathbf{A}^{n}\right)^{G}$ over $k$ follows from Corollary 7 and Lemma 5.

Remark 4. A weakened version of Theorem 10 is the subject of [Vin92]. In it, $G$ is an affine algebraic group and $\widehat{\mathcal{J}}\left(x_{1}, \ldots, x_{n}\right)$ is replaced by $\mathcal{J}\left(x_{1}, \ldots\right.$ $\left.\ldots, x_{n}\right)$. However, the argument in [Vin92] does not amount to complete and accurate proof. Indeed, it is based on the claim, left unproven, that if $G$ is reductive, then $G$ is conjugate in $\mathcal{J}\left(x_{1}, \ldots, x_{n}\right)$ to a subgroup of $D_{n}$. Further, the claim that, for a one-dimensional unipotent algebraic group $U$, "every point is $U$-equivalent to a unique point of the subspace $S=\left\{x \in k^{n}: x_{m}=\right.$ $0\}$ " is false because $u \cdot s$ may be not defined for $u \in U$ and $s \in S$. Ditto for the claim that $F_{i} \in k\left(x_{i+1}, \ldots, x_{n}\right) \otimes k[t]$ (counterexample: $n=3$ and the action is given by $\left.t \cdot x_{1}=x_{1}-t / x_{2}\left(x_{2}+t\right), t \cdot x_{2}=x_{2}+t, t \cdot x_{3}=x_{3}\right)$, so the equality $F_{m}\left(x_{m+1}, \ldots, x_{n} ; t\right)=t F_{m}\left(x_{m+1}, \ldots, x_{n}\right)$ remains unproven.

Remark 5 . One cannot replace $\widehat{\mathcal{J}}\left(t_{1}, \ldots, t_{n}\right)$ in Theorem 10 by the $\mathcal{C}_{n^{-}}$ stabilizer of the flag of subfields (20). Indeed, by [Tri80], for $k=\mathbf{C}, n=3$, this stabilizer contains a subgroup $G$ of order 2 such that $k\left(\mathbf{A}^{n}\right)^{G}$ is not pure over $k$. 
Combining the construction from [Tri80] with Corollary 3 and Lemma 5 we obtain the following

Theorem 11. Let $k=\mathbf{C}$ and let $\mathcal{A}$ be the union of all connected affine algebraic subgroups of $\mathcal{C}_{\infty}$. There exists an element $g \in \mathcal{C}_{3}$ of order 2 such that $g \notin \mathcal{A}$. In particular, $g$ is not stably linearizable.

Proof. Let $X$ be the three-dimensional counterexample of Artin and Mumford to the Lüroth problem ([AM72], see also [Del70]): $X$ is a smooth projective unirational threefold such that

$$
\mathrm{H}^{3}(X, \mathbf{Z})_{\text {tors }} \neq 0 \text {. }
$$

Since the torsion subgroup of the third integral cohomology group of a smooth complex variety is a birational invariant and, in particular, is zero if the variety is rational, (47) implies that $X$ is not rational.

In [Tri80] is constructed a subgroup $G$ of order 2 in $\mathcal{C}_{3}$ such that $k\left(\mathbf{A}^{3}\right)^{G}$ is $k$-isomorphic to $k(X)$. Let $g$ be the generator of $G$. Arguing on the contrary, assume that $g$ is contained in a connected affine algebraic subgroup $H$ of $\mathcal{C}_{\infty}$. Since the order of $g$ is finite, $g$ is a semisimple element of $H$. Hence, $g$ lies in a maximal torus $T$ of $H$ (see [Bor91, Theorems III.10.6(6) and IV.11.10]). By Corollary 3 there exists a positive integer $n_{0}$ such that $T \subset$ $\mathcal{C}_{n_{0}}$ and $T$ is conjugate in $\mathcal{C}_{n_{0}}$ to a subtorus of $D_{n_{0}}$. Fix an integer $n \geqslant$ $\max \left\{n_{0}, 3\right\}$. Then $G \subset \mathcal{C}_{n}$ and $G$ is conjugate in $\mathcal{C}_{n}$ to a subgroup of $D_{n}$. This and Lemma 5 yield that for the natural action of $G$ on $\mathbf{A}^{n}$ the field $k\left(\mathbf{A}^{n}\right)^{G}$ is pure over $k$. Since $G \subset \mathcal{C}_{3}$, by [Ros61 $1_{2}$, Lemma 3], we have

$$
\mathbf{A}^{n}{ }_{i}^{\prime} G \approx \mathbf{A}^{3} ; G \times \mathbf{A}^{n-3} \approx X \times \mathbf{P}^{n-3}
$$

From (48) we infer that the smooth projective variety $X \times \mathbf{P}^{n-3}$ is rational and therefore $\mathrm{H}^{3}\left(X \times \mathbf{P}^{n-3}\right)_{\text {tors }}=0$. On the other hand, the Künneth formula and (47) yield that $\mathrm{H}^{3}\left(X \times \mathbf{P}^{n-3}\right)_{\text {tors }} \neq 0-$ a contradiction.

We conclude by an example which shows that in the formulation of Corollary 8 "unipotent" cannot be replaced by "connected solvable" (recall that if $G$ is connected solvable, then the existence of some rational cross-section is ensured by [Ros56, Theorem 10]).

Example 7. Fix a choice of two integers $d_{1}$ and $d_{2}$ such that

$$
\begin{gathered}
d_{1}-d_{2} \geqslant 2, \\
\left|d_{1}\right| \geqslant 2, \quad\left|d_{2}\right| \geqslant 2, \\
\operatorname{gcd}\left(d_{1}, d_{2}\right)=1 .
\end{gathered}
$$

Consider the one-dimensional subtorus

$$
T:=\left\{\operatorname{diag}\left(t^{d_{1}}, t^{d_{2}}\right) \mid t \in k^{*}\right\}
$$

of $D_{2}$ and its rational linear action $\alpha$ on $\mathbf{A}^{2}$ defined by formula (4).

In view of (51), the $T$-stabilizer of every point $a \in \mathbf{A}^{2}, a \neq(0,0)$, is trivial.

Claim. There is no affine subspace in $\mathbf{A}^{2}$ that is a rational cross-section for $\alpha$. 
Proof. Assume that some affine subspace $L$ of $\mathbf{A}^{2}$ is a rational cross-section for $\alpha$. Since $T$-orbits in general position are one-dimensional, $L$ is a line. Let

$$
\mu_{1} x_{1}+\mu_{2} x_{2}+\nu=0, \quad \mu_{1}, \mu_{2}, \nu \in k .
$$

be its equation. Since $L$ is a rational cross-section, there is a nonempty open subset $U$ of $\mathbf{A}^{2}$ such that for every point $a=\left(a_{1}, a_{2}\right) \in U$, the $T$-orbit of $a$ intersects $L$ at a single point, i.e., by (52) and (53), the following equation in $t$

$$
\mu_{1} a_{1} t^{d_{1}}+\mu_{2} a_{2} t^{d_{2}}+\nu=0
$$

has a single nonzero solution. Shrinking $U$, we may assume that $a_{1} a_{2} \neq 0$ for every $a \in U$.

If $\mu_{1} \mu_{2}=0$, then (54) becomes an equation of the form $\mu t^{d}+\nu=0$ where $\mu \in k, \mu \neq 0$, and $|d| \geqslant 2$ by (50). If $\nu=0$, it does not have nonzero solutions; if $\nu \neq 0$, there are at least two such solutions. So this case is impossible.

If $\mu_{1} \mu_{2} \neq 0$ and $\nu=0$, then the solutions of (54) coincide with the roots of $\mu_{1} a_{1} t^{d_{1}-d_{2}}+\mu_{2} a_{2}$. In view of (49), there are at least two distinct roots, so this case is impossible as well.

Let $\mu_{1} \mu_{2} \nu \neq 0$ and $d_{2}>0$. Denote by $f$ be the right-hand side of (54). Set

$$
h:=d_{1} f-t \frac{d f}{d t}=\left(d_{1}-d_{2}\right) \mu_{2} a_{2} t^{d_{2}}+d_{1} \nu
$$

By (54) and (55), for a fixed $a_{2}$, there are only finitely many $a_{1}$ 's such that the polynomials $f$ and $h$ have a common root. Since every multiple root of $f$ is also a root of $h$, this means that there are points $a \in U$ such that $f$ does not have multiple roots. From (49), (50) it then follows that for such a point $a$ equation (54) has at least two nonzero solutions. Thus, this case is also impossible.

Finally, let $\mu_{1} \mu_{2} \nu \neq 0$ and $d_{2}<0$. Then the solutions of equation (54) coincide with the roots of the polynomial $q:=\mu_{1} a_{1} t^{d_{1}-d_{2}}+\nu t^{-d_{2}}+\mu_{2} a_{2}$. We have

$$
p:=\left(d_{1}-d_{2}\right) q-t \frac{d q}{d t}=\left(d_{1}-d_{2}\right) \mu_{2} a_{2}+d_{1} \nu t^{-d_{2}} .
$$

Then the same argument as above with $f$ and $h$ replaced resp. by $q$ and $p$ shows that this case is impossible as well.

This contradiction completes the proof.

\section{REFERENCES}

[AHK00] H. Ahmad, M. HajJA, M.-C. KAng, Rationality of some projective linear actions, J. Algebra 228 (2000), 643-658.

[AM72] M. Artin, D. Mumford, Some elementary examples of unirational varieties which are not rational, Proc. London Math. Soc. (3) 25 (1972), 75-95.

[BCSS85] A. Beauville, J.-L. Colliot-Thélène, J.-J. Sansuc, P. SwinnertonDyeR, Varietes stablement rationneles non rationneles, Annals of Math. 121 (1985), no. 2, 283-318.

[Bia66] A. BiAŁYNiCKI-BiRula, Remarks on the action of an algebraic torus on $k^{n}$, Bull. Acad. Polon. Sci. Sér. Sci. Math., Astr., Phys. 14 (1966), no. 4, 177-181.

[Bor91] A. Borel, Linear Algebraic Groups, Graduate Texts in Mathematics, Vol. 126, 2nd edn. (Springer-Verlag, New York, 1991). 
[BS64] A. Borel, J.-P. SERre, Théorèmes de finitude en cohomogie galoisienne, Comm. Math. Helv. 39 (1964), 111-164.

[Che54] C. Chevalley, On algebraic group varieties, J. Math. Soc. Japan 6 (1954), nos. 3-4, 303-324.

[CTKPR11] J.-L. Colliot-Thélène, B. Kunyavskǐ̌, V. L Popov, Z. Reichstein, Is the function field of a reductive Lie algebra purely transcendental over the field of invariants for the adjoint action?, Compositio Math. 147 (2011), no. 02, 428-466.

[Del70] P. Deligne, Variétés unirationnelles non rationnelles (d'après M. Artin et D. Mumford), in Séminaire Bourbaki, Lecture Notes in Mathematics, Vol. 317, Exp. no. 402 (Springer-Verlag, Berlin, 1973), pp. 45-57.

[Dem70] M. Demazure, Sous-groupes algébriques de rang maximum du groupe de Cremona, Ann. Sci. de l'E.N.S., 4e sér. 3 (1970), fasc. 4, 507-588.

[Gro58] A. GRothENDIECK, Torsion homologique et sections rationnelle, in Anneaux de Chow et Applications, Séminaire Claude Chevalley, Vol. 3, Exp. no. 5 (Secrétariat mathématique, Paris, 1958), pp.1-29.

[Hal59] M. HALl, JR., The Theory of Groups (Macmillan, New York, 1959).

[Hir72] A. Hinschowitz, Le groupe de Cremona d'après Demazure, in Séminaire N. Bourbaki, Lecture Notes in Mathematics, Vol. 317, Exp. no. 413 (SpringerVerlag, Berlin, 1973), pp. 261-276.

[Jos11] A. Joseph, An algebraic slice in the coadjoint space of the Borel and the Coxeter element, Adv. Math. 227 (2011), no. 1, 522-585.

[KV89] M. KervaIRE, T. Vust, Fractions rationnelles invariantes par un groupe fini: Quelques exemples, in: Algebraic Transformation Gropus and Invariant Theory, DMV Seminar, Band 13, Birkhäuser, Basel, 1989, pp. 157-179.

[Kos63] B. Kostant, Lie group representations on polynomial rings, Amer. J. Math. 85 (1963), 327-404.

[Mer80] Y. I. Merzlyakov, Rational Groups (Nauka, Moscow, 1980) (in Russian).

[Miy71] T. MiYata, Invariants of certain groups I, Nagoya Math. J. 41 (1971), 69-73.

[Pop72] V. L. Popov, On the stability of the action of an algebraic group on an algebraic variety, Math. USSR Izv. 6 (1972), 367-379 (1973).

[Pop94] V. L. Popov, Sections in invariant theory, in Proceedings of The Sophus Lie Memorial Conference, Oslo 1992 (Scandinavian University Press, Oslo, 1994), pp. 315-362.

[PV94] V. L. Popov, E. B. VinberG, Invariant theory, in Algebraic Geometry IV, Encyclopaedia of Mathematical Sciences, Vol. 55 (Springer-Verlag, Berlin, 1994), pp. 123-284.

[Pu] L. Pukanszky, Leçns sur les Représentations des Groupes, Monographies de la Société Mathématique de France, Vol. 2 (Dunod, Paris, 1967).

[Ros56] M. Rosenlicht, Some basic theorems on algebraic groups, Amer. J. Math. 78 (1956), 401-443.

[Ros61 1 M. Rosenlicht, Toroidal algebraic groups, Proc. Amer. Math. Soc. 12 (1961), 984-988.

[Ros61 2 M. RosEnLICHT, On quotient variaties and the affine embedding of certain homogeneous spaces, Trans. Amer. Math. Soc. 101 (1961), 211-223.

[Ser58] J.-P. SERRE, Espaces fibrés algébriques, in Anneaux de Chow et Applications, Séminaire Claude Chevalley, Vol.3, Exp.no.1 (Secrétariat mathématique, Paris, 1958), pp. 1-37.

[Ser08] J.-P. SERRE, Le groupe de Cremona et ses sous-groupes finis, in Séminaire N. Bourbaki, Vol. 2008/2009, Astérisque Vol. 332, Exp. no. 1000 (Société Mathématique de France, 2010), pp. 75-100.

[Sh-B04] N.-I. ShEPhERD-BARRon, Stably rational irrational varieties, in: The Fano Conference, Univ. Torino, Turin, 2004, pp. 693-700.

[Sum74] H. Sumihiro, Equivariant completion, J. Math. Kyoto Univ. 14 (1974), no. 1, $1-28$. 
[Tri80] D. D. TRIANTAPhyllou, Invariants of finite groups acting nonlinearly on rational function fields, J. Pure Appl. Algebra 18 (1980), 315-331.

[vdE00] A. VAn Den Essen, Polynomial Automorphisms, Progress in Mathematics, Vol. 190 (Birkhäuser, Basel, 2000).

[vdW67] B. L. van der WAerden, Algebra I (Springer-Verlag, Berlin, 1967).

[Vin92] E. B. VinBerG, Rationality of the field of invariants of a triangular group, Mosc. Univ. Math. Bull. 37 (1992), no. 2, 27-29.

[Wei55] A. WEIL, On algebraic groups of transformations, Amer. J. Math. 77 (1955), no. $2,355-391$.

Steklov Mathematical Institute, Russian Academy of Sciences, Gubkina 8, Moscow, 119991, Russia

E-mail address: popovvl@mi.ras.ru 